\title{
Propriedades da expansão decimal
}

Fernanda Martinez Menezes 

Data de Depósito:

Assinatura:

\title{
Fernanda Martinez Menezes
}

\section{Propriedades da expansão decimal}

\begin{abstract}
Dissertação apresentada ao Instituto de Ciências Matemáticas e de Computação - ICMC-USP, como parte dos requisitos para obtenção do título de Mestra - Programa de Mestrado Profissional em Matemática. EXEMPLAR DE DEFESA
\end{abstract}

Área de Concentração: Matemática

Orientador: Prof. Dr. Benito Frazão Pires

\section{USP - São Carlos}

Novembro de 2015 
Ficha catalográfica elaborada pela Biblioteca Prof. Achille Bassi

e Seção Técnica de Informática, ICMC/USP com os dados fornecidos pelo(a) autor(a)

M543p $\begin{aligned} & \text { Menezes, Fernanda Martinez } \\ & \text { Propriedades da expansão decimal / Fernanda } \\ & \text { Martinez Menezes; orientador Benito Frazão Pires. } \\ & \text { - São Carlos - SP, 2015. } \\ & 45 \text { p. } \\ & \text { Dissertação (Mestrado - Programa de Pós-graduação } \\ & \text { em Mestrado Profissional em Matemática em Rede } \\ & \text { Nacional) - Instituto de Ciências Matemáticas e de } \\ & \text { Computação, Universidade de São Paulo, 2015. } \\ & \text { 1. Expasão decimal. 2. números racionais e } \\ & \text { irracionais. 3. dígitos. I. Pires, Benito Frazão, } \\ & \text { orient. II. Título. }\end{aligned}$




\title{
Fernanda Martinez Menezes
}

\section{Properties of the decimal expansion}

\begin{abstract}
Master dissertation submitted to the Instituto de Ciências Matemáticas e de Computação - ICMC-USP, in partial fulfillment of the requirements for the degree of the Master - Program in Mathematics Professional Master. EXAMINATION BOARD PRESENTATION COPY
\end{abstract}

Concentration Area: Mathematics

Advisor: Prof. Dr. Benito Frazão Pires

USP - São Carlos

November 2015 

Primeiramente agradeço meu marido Pedro Henrique, meu principal incentivador e pessoa presente desde a prova de seleção do PROFMAT até a defesa final desse trabalho. Não tenho palavras para descrever como seu apoio e companheirismo foram importantes para que trabalho tivesse chegado ao fim.

Agradeço também a todos da minha família que me apoiaram ao longo dessa empreitada. Em especial, a minha mãe Carmem, que sempre estimulou a minha carreira de docente nessa maravilhosa disciplina que é a Matemática. É um orgulho poder trilhar mais esse caminho seguindo o seu maior conselho: todos nós podemos nos tornar melhores naquilo que fazemos nos aperfeiçoando e atualizando os nossos conhecimentos cada vez mais.

Agradeço a todos os meus colegas da turma de Mestrado, em especial, aqueles com os quais dividi carona em diversos sábados até as aulas. Nesses $90 \mathrm{kms}$ de viagem, trocamos experiências, alegrias, tristezas, mas principalmente, um apoio incondicional para nosso objetivo final pudesse ser alcançado.

Um agradecimento a todos os meus professores do curso, por me transmitirem seus conhecimentos e contribuírem para o meu aperfeiçoamento profissional. Em especial, quero agradecer a professora e coordenadora do PROFMAT, Kátia, que, nos meus momentos de maior insegurança e desespero, sempre me acalmava e me auxiliava com o que precisava.

Agradeço ao meu orientador Benito, por sua dedicação e a CAPES pelo indispensável apoio financeiro ao longo dessa pós-graduação.

E agradeço a Deus, acima de tudo. 



\section{RESUMO}

MENEZES, F. M.. Propriedades da expansão decimal. 2015. 45 f. Dissertação (Mestrado Programa de Mestrado Profissional em Matemática) - Instituto de Ciências Matemáticas e de Computação (ICMC/USP), São Carlos - SP.

Este trabalho tem como objetivo principal o estudo da expansão decimal dos números reais. Primeiramente provamos que todo número real possui ao menos uma expansão decimal. $\mathrm{Na}$ sequência, um método para encontrar a expansão decimal de um número entre 0 e 1 é apresentado, bem como um estudo sobre a expansão decimal de números racionais e irracionais. Em seguida, o estudo apresenta métodos que permitem encontrar aproximações racionais de números irracionais, além dos erros cometidos por essas aproximações. Na parte final, por seu turno, o foco do trabalho recai sobre a análise da regularidade (frequência) dos dígitos das expansões decimais.

Palavras-chave: Expasão decimal, números racionais e irracionais, dígitos. 



\section{ABSTRACT}

MENEZES, F. M.. Propriedades da expansão decimal. 2015. 45 f. Dissertação (Mestrado Programa de Mestrado Profissional em Matemática) - Instituto de Ciências Matemáticas e de Computação (ICMC/USP), São Carlos - SP.

This work has as main objective the study of the decimal expansion of the real numbers. First we prove that every real number has at least one decimal expansion. Further, a method to find the decimal expansion of real numbers between 0 and 1 is provided as well as a the study of the decimal expansion of rational and irrational numbers. Next, the study presents methods that provide rational approximations to irrational numbers, in addition to the errors committed by these approximations. At the end, by its turn, the focus of the work is put on the analysis of the regularity (frequency) of the digits of the decimal expansion.

Key-words: decimal expansion, rational and irrational numbers, digits. 



\section{LISTA DE ILUSTRAÇÕES}

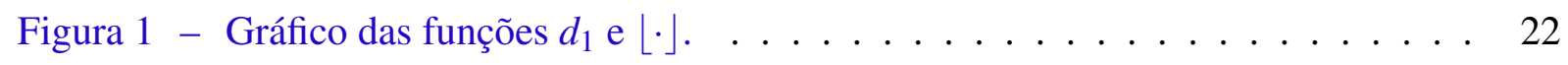

Figura $2-$ Gráfico da função $f \ldots \ldots \ldots \ldots \ldots \ldots$ 



\section{LISTA DE TABELAS}

Tabela 1 - Tabela com as primeiras 21 iterações do Método Babilônico para $\sqrt{2}$. . . . 33

Tabela 2 - Tabela com as primeiras 22 iterações do Método Babilônico para $\sqrt{3}$. . . . 34

Tabela 3 - Tabela com as primeiras 21 iterações do Método Babilônico para $\sqrt{17}$. . . 35 

INTRODUÇÃO . . . . . . . . . . . . . . . . . 17

A EXPANSÃO DECIMAL . . . . . . . . . . . . . . . . . . . . . 19

2.1 Séries de termos não-negativos . . . . . . . . . . . . . . 19

2.1.1 Existência da expansão decimal . . . . . . . . . . . . . . . . . . 20

2.1.2 Algoritmo para calcular a expansão decimal . . . . . . . . . . . . 22

2.2 Expansão decimal dos números racionais . . . . . . . . . . . . 25

2.3 Números com expansão decimal finita . . . . . . . . . . . . . 27

$2.4 \quad$ Unicidade da expansão decimal . . . . . . . . . . . . . 28

$2.5 \quad$ Expansão decimal de números irracionais quadráticos . . . . . . . 30

$2.6 \quad 0$ Método Babilônico para encontrar $\sqrt{p} \ldots \ldots \ldots$

2.6.1 Outra prova de que $\lim _{n \rightarrow \infty} x_{n}=\sqrt{p} \ldots \ldots \ldots \ldots$

$2.7 \quad$ Outras abordagens do Método Babilônico . . . . . . . . . . . 35

3 REGULARIDADE DOS DÍGITOS DA EXPANSÃO DECIMAL . . . 41

3.1 Conjuntos de medida zero e de medida total . . . . . . . . . . 41

$3.2 \quad$ Números simplesmente normais . . . . . . . . . . . . . . . 41 

Os elementos do conjunto $\{0,1,2, \ldots, 9\}$ são chamados dígitos. O termo dígito vem do latim digitum ("dedo") e está relacionado com o fato de que os dedos das mãos correspondem aos 10 símbolos do sistema de numeração decimal ou em base 10 .

Todo número $x \in[0,1)$ pode ser representado como uma sequência infinita de dígitos $d_{1}, d_{2}, \ldots$ chamada expansão decimal de $x$, a qual é denotada por $x=0, d_{1} d_{2}, \ldots$ Quando $x \in[0,1)$ é um número racional, a expansão decimal de $x$ é periódica e pode ser obtida através de um número finito de operações. Neste caso, é possível exibir o histograma da expansão decimal de $x$, isto é, é possível calcular exatamente a frequência $\mathbb{P}(d)$ com que cada dígito $d$ aparece na expansão decimal de $x$ :

$$
\mathbb{P}(d)=\lim _{n \rightarrow \infty} \frac{\#\left\{1 \leq k \leq n \mid d_{k}=d\right\}}{n},
$$

onde \# denota a cardinalidade do conjunto. Por exemplo,

$$
\frac{12.347}{99.990}=0,12348234823482348 \ldots
$$

A expansão decimal de $x$ é finita se existir um número $n \geq 1$ tal que $d_{k}=0$ para todo $k>n$. Neste caso, todos os dígitos diferentes de zero aparecem com frequência 0 , enquanto o dígito 0 aparece com frequência $100 \%$. Por exemplo,

$$
\frac{1}{20}=0,05=0,05000 \ldots
$$

Assim, $\mathbb{P}(0)=100 \%$ e $\mathbb{P}(d)=0$ para todo $d \neq 0$.

Quando $x$ é um número irracional, a expansão decimal de $x$ não é periódica e o padrão com que os dígitos aparecem pode ser bastante irregular. Por exemplo, os primeiros 40 dígitos da expansão decimal de $\frac{\sqrt{2}}{2}$ são dados por:

$$
\frac{\sqrt{2}}{2}=0.7071067811865475244008443621048490392848 \ldots
$$


Apesar da irregularidade no padrão observado na expansão dos números irracionais, há um fenômeno bastante interessante que ocorre com a maior parte dos números irracionais. Dizemos que um número $x \in[0,1)$ é simplesmente normal se a frequência $\mathbb{P}(d)$ com que cada dígito aparece é a mesma para qualquer dígito $d \in\{0,1, \ldots, 9\}$. Assim, $x \in[0,1)$ é simplesmente normal se

$$
\mathbb{P}(d)=10 \% \quad \text { para todo } \quad d \in\{0,1, \ldots, 9\} .
$$

Este texto inclui um resultado (Teorema 12) que afirma que a maior parte dos números reais é simplesmente normal.

Todos os resultados apresentados acima serão abordados com detalhes nas próximas seções. Esta obra não contém nenhum resultado original. E. Borel provou em 1909 um resultado que inclui o Teorema 12 como um caso particular. O algoritmo (Teorema 1) apresentado aqui para gerar a expansão decimal de um número $x \in[0,1)$ usa a abordagem da área de Sistemas Dinâmicos, que estuda as propriedades dos iterados $f, f^{2}, f^{3}, \ldots$ de uma função $f: X \rightarrow X$. Neste caso, temos que $X=[0,1)$ e $f(x)=\{10 x\}$, onde \{\} denota a parte fracionária de um número. Esta abordagem pode ser encontrada em [2] . A prova da existência da expansão decimal tal qual apresentada aqui pode ser encontrada em [2], enquanto a prova da unicidade da expansão decimal é baseada em [4]. Também foi incluída nesta obra métodos numéricos para encontrar a expansão decimal de um número irracional quadrático $x$. Uma das abordagens apresentada aqui usa contrações e o Teorema do Ponto Fixo de Banach. A estimativa do erro de aproximação (Teorema 10) pode ser encontrada em [3]. 
Dizemos que uma sequência infinita de dígitos $d_{1}, d_{2}, \ldots$ é uma expansão decimal do número $x \in[0,1)$ se a série $\sum_{k=1}^{\infty} d_{k} 10^{-k}$ for convergente e tiver soma igual a $x$. Nesse caso, escrevemos:

$$
x=0, d_{1} d_{2} \ldots=\frac{d_{1}}{10}+\frac{d_{2}}{10^{2}}+\cdots .
$$

O primeiro aspecto que deve ser considerado acerca da expansão decimal de um número é a convergência de uma série da forma $\sum_{k=1}^{\infty} d_{k} 10^{-k}$. Trata-se de uma śerie de termos nãonegativos pois $d_{k} \geq 0$ para todo $k \geq 1$. Este aspecto é abordado na próxima seção.

\subsection{Séries de termos não-negativos}

Seja $\left(s_{n}\right)_{n=1}^{\infty}$ uma sequência de números reais. Dizemos que $\left(s_{n}\right)$ é crescente (respectivamente, decrescente) se $s_{n} \leq s_{n+1}$ (respectivamente, $s_{n} \geq s_{n+1}$ ) para todo $n \geq 1$. Dizemos que $\left(s_{n}\right)$ é limitada superiormente se existir um número $M$ tal que $s_{n} \leq M$ para todo $n \geq 1$. Dizemos que $\left(s_{n}\right)$ é limitada inferiormente se existir um número $M$ tal que $s_{n} \geq M$ para todo $n \geq 1$. Dizemos que $\left(s_{n}\right)$ converge para a se para todo $\varepsilon>0$, existir um número natural $N$ tal que $\left|s_{n}-a\right|<\varepsilon$ para todo $n \geq N$. A sequência $\left(s_{n}\right)$ é dita convergente se ela convergir para algum número $a$. Neste caso, o número $a$ é único e é chamado de limite de $s_{n}\left(\lim _{n \rightarrow \infty} s_{n}=a\right)$.

\section{Lema 1.}

(a) Toda sequência crescente e limitada superiormente é convergente.

(b) Toda sequência decrescente e limitada inferiormente é convergente.

Demonstração. (a) Seja $\left(s_{n}\right)_{n=1}^{\infty}$ uma sequência crescente e limitada superiormente. Então o conjunto $\left\{s_{n}: n \geq 1\right\}$ tem um supremo $s \in \mathbb{R}$. Afirmamos que $\lim _{n \rightarrow \infty} s_{n}=s$. De fato, pela definição de $s$, para cada $\varepsilon>0$, existe um elemento $s_{N}$ tal que $s_{N}>s-\varepsilon$. Como $\left(s_{n}\right)$ é crescente, 
isto implica que $s-\varepsilon<s_{N} \leq s_{n} \leq s<s+\varepsilon$ para todo $n \geq N$. Isto é, $\left|s_{n}-s\right|<\varepsilon$ para todo $n \geq N$. Isto mostra que $\lim _{n \rightarrow \infty} s_{n}=s$. (b) A prova destes caso é feita de maneira análoga ao caso $(a)$.

Proposição 1. Para toda sequência de dígitos $d_{1}, d_{2}, \ldots$, a série $\sum_{k=1}^{\infty} d_{k} 10^{-k}$ é convergente e sua soma é um número do intervalo $[0,1]$.

Demonstração. Para provar que a série $\sum_{k=1}^{\infty} d_{k} 10^{-k}$ é convergente, devemos estudar a sequência $\left(s_{n}\right)_{n=1}^{\infty}$ formada pelas somas parciais $s_{n}=\sum_{k=1}^{n} d_{k} 10^{-k}$ da série. Esta sequência é crescente pois $s_{n+1}=s_{n}+\frac{d_{n+1}}{10^{n+1}} \geq s_{n}$ para todo $n \geq 1$. Vamos mostrar agora que $\left(s_{n}\right)$ é limitada superiormente por 1 e inferiormente por 0 . De fato, como $d_{k} \leq 9$, temos que

$$
0 \leq s_{n}=\sum_{k=1}^{n} \frac{d_{k}}{10^{k}} \leq \sum_{k=1}^{n} \frac{9}{10^{k}}<\frac{9}{10}\left(1+\frac{1}{10}+\frac{1}{10^{2}}+\cdots\right)=\frac{9}{10} \frac{1}{1-\frac{1}{10}}=1
$$

Pelo Lemma $1,\left(s_{n}\right)$ e, portanto, $\sum_{k=1}^{\infty} d_{k} 10^{-k}$, converge para algum número do intervalo $[0,1]$.

\subsubsection{Existência da expansão decimal}

Proposição 2. Todo número real $x \in[0,1)$ admite pelo menos uma expansão decimal.

Demonstração. Seja $x \in[0,1)$ dado. Primeiramente, vamos definir por indução uma sequência $d_{1}, d_{2} \ldots$ de dígitos satisfazendo algumas propriedades. O primeiro dígito $d_{1}$ é definido por

$$
d_{1}=\max \left\{j \mid 0 \leq j \leq 9 \mathrm{e} \frac{j}{10} \leq x\right\}
$$

Note que, por definição,

$$
\frac{d_{1}}{10} \leq x<\frac{d_{1}}{10}+\frac{1}{10}
$$

Vamos proceder por indução para definir os dígitos seguintes: $d_{2}, d_{3}, \ldots$. Suponha que os $n$ primeiros dígitos $d_{1}, d_{2}, \ldots, d_{n}$ tenham sido definidos e que

$$
\frac{d_{1}}{10}+\frac{d_{2}}{10^{2}}+\cdots+\frac{d_{n}}{10^{n}} \leq x<\frac{d_{1}}{10}+\frac{d_{2}}{10^{2}}+\cdots+\frac{d_{n}}{10^{n}}+\frac{1}{10^{n}} .
$$

Note que (2.4) é verdadeira quando $n=1$. O dígito $d_{n+1}$ é definido por

$$
d_{n+1}=\max \left\{j \mid 0 \leq j \leq 9 \text { e } \frac{d_{1}}{10}+\frac{d_{2}}{10^{2}}+\ldots+\frac{d_{n}}{10^{n}}+\frac{j}{10^{n+1}} \leq x\right\} .
$$

Afirmamos que

$$
\frac{d_{1}}{10}+\frac{d_{2}}{10^{2}}+\cdots+\frac{d_{n}}{10^{n}}+\frac{d_{n+1}}{10^{n+1}} \leq x<\frac{d_{1}}{10}+\frac{d_{2}}{10^{2}}+\cdots+\frac{d_{n}}{10^{n}}+\frac{d_{n+1}}{10^{n+1}}+\frac{1}{10^{n+1}}
$$


De fato, a primeira desigualdade em (2.6) segue imediatamente da definição de $d_{n+1}$. A segunda desigualdade pode ser provada por absurdo. Suponha que ela é falsa, isto é, que

$$
\frac{d_{1}}{10}+\frac{d_{2}}{10^{2}}+\cdots+\frac{d_{n}}{10^{n}}+\frac{d_{n+1}}{10^{n+1}}+\frac{1}{10^{n+1}} \leq x .
$$

Dois casos devem ser considerados: (a) $d_{n+1}=9$ ou (b) $d_{n+1}<9$. No caso (a), (2.7) implica que

$$
\frac{d_{1}}{10}+\frac{d_{2}}{10^{2}}+\cdots+\frac{d_{n}}{10^{n}}+\frac{1}{10^{n}}=\frac{d_{1}}{10}+\frac{d_{2}}{10^{2}}+\cdots+\frac{d_{n}}{10^{n}}+\frac{d_{n+1}}{10^{n+1}}+\frac{1}{10^{n+1}} \leq x,
$$

o que contraria a segunda desigualdade em (2.4). No caso (b), $d_{n+1}+1 \in\{1, \ldots, 9\}$ e

$$
\frac{d_{1}}{10}+\frac{d_{2}}{10^{2}}+\cdots+\frac{d_{n}}{10^{n}}+\frac{d_{n+1}+1}{10^{n+1}}=\frac{d_{1}}{10}+\frac{d_{2}}{10^{2}}+\cdots+\frac{d_{n}}{10^{n}}+\frac{d_{n+1}}{10^{n+1}}+\frac{1}{10^{n+1}} \leq x,
$$

contrariando a definição de $d_{n+1}$.

Por indução, concluímos que a sequência de dígitos $d_{1}, d_{2}, \ldots$, definida acima satisfaz (2.4) para todo $n \geq 1$.

Para terminarmos a prova da proposição, precisamos mostrar que a sequência de dígitos $d_{1}, d_{2}, \ldots$ é, de fato, a expansão decimal de $x$, isto é, que $x=\lim _{n \rightarrow \infty} \sum_{k=1}^{n} d_{k} 10^{-k}$.

Segue de (2.4) que

$$
\left|\sum_{k=1}^{n} \frac{d_{k}}{10^{k}}-x\right|<\frac{1}{10^{n}}, \quad \text { portanto } \quad x=\lim _{n \rightarrow \infty} \sum_{k=1}^{n} d_{k} 10^{-k}
$$

Observação 1. A sequência de dígitos $d_{1}, d_{2}, \ldots$ definida pelas equações (2.2) e (2.5) não termina numa sequência infinita de 9's, isto é, não existe $r \geq 1$ tal que $d_{k}=9$ para todo $k \geq r$. Em particular,

$$
\sum_{k=1}^{\infty} \frac{d_{k+r}}{10^{k}}<1 \quad \text { para todo } \quad r \geq 1
$$

Demonstração. Assuma que exista $r \geq 1$ tal que $d_{k}=9$ para todo $k \geq r$. Observe que $r>1$, caso contrário teríamos $x=\sum_{k=1}^{\infty} 9 \cdot 10^{-k}=1 \notin[0,1)$. Assim, podemos supor que $r \geq 2$. Então,

$$
x=\sum_{k=1}^{\infty} \frac{d_{k}}{10^{k}}=\sum_{k=1}^{r-1} \frac{d_{k}}{10^{k}}+\sum_{k=r}^{\infty} \frac{9}{10^{k}}=\sum_{k=1}^{r-1} \frac{d_{k}}{10^{k}}+\frac{9}{10^{r}}\left(\frac{1}{1-\frac{1}{10}}\right)=\sum_{k=1}^{r-1} \frac{d_{k}}{10^{k}}+\frac{1}{10^{r-1}} .
$$

Isto contradiz a segunda desigualdade em (2.4), que afirma que $x<\sum_{k=1}^{r-1} \frac{d_{k}}{10^{k}}+\frac{1}{10^{r-1}}$. Isto prova a primeira parte da observação. Assim, para todo $r \geq 1$, existe algum $m \geq r$ tal que $d_{m}<9$. Isto garante que a desigualdade abaixo é estrita:

$$
\sum_{k=1}^{\infty} \frac{d_{k+r}}{10^{k}}<\sum_{k=r}^{\infty} \frac{9}{10^{k}}=1
$$




\subsubsection{Algoritmo para calcular a expansão decimal}

A sequência de dígitos $d_{1}, d_{2}, \ldots$ definida pelas equações (2.2) e (2.5) pode ser considerada uma sequência de funções de $x$. Neste sentido, vamos denotar por $d_{k}(x)$ o $k$-ésimo dígito da expansão decimal de $x$. Um gráfico da função $d_{1}:[0,1) \rightarrow\{0,1, \ldots, 9\}$ é dada na Figura 1 . Na mesma figura está esboçado o gráfico da função máximo inteiro $\lfloor\cdot\rfloor: \mathbb{R} \rightarrow \mathbb{Z}$ definida por:

$$
\lfloor y\rfloor=\max \{k \in \mathbb{Z} \mid k \leq y\}, \quad y \in \mathbb{R} .
$$

Figura 1 - Gráfico das funções $d_{1}$ e $\lfloor\cdot \cdot$.
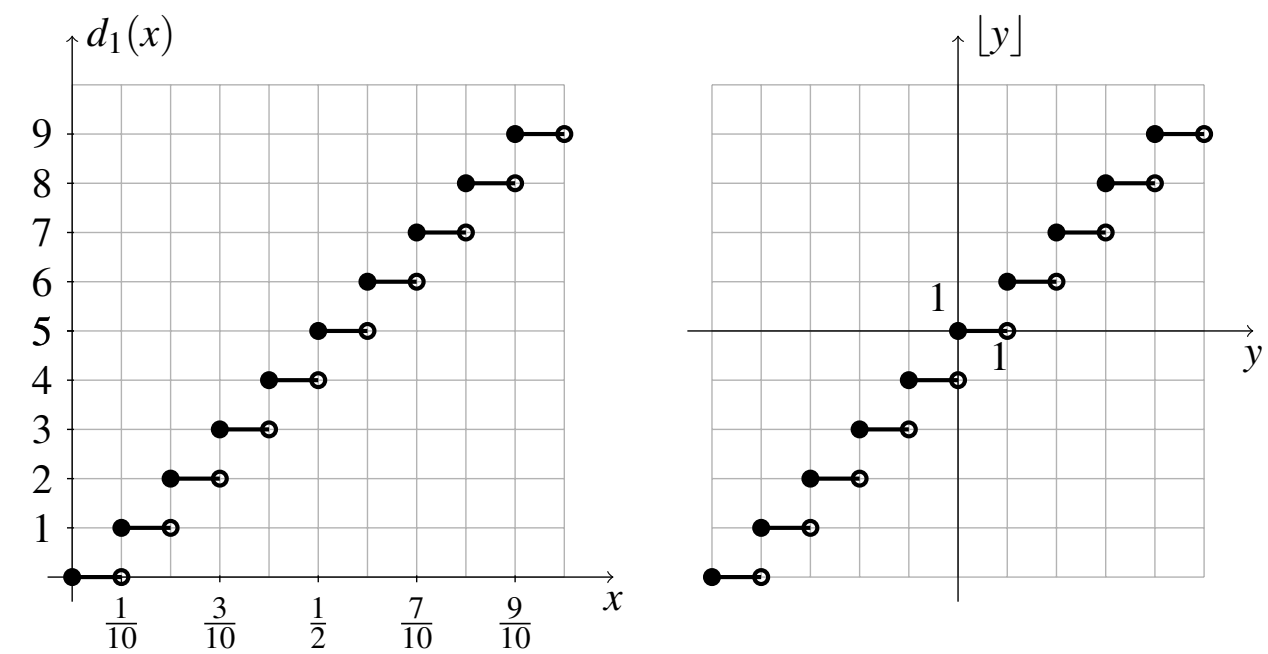

Segue imediatamente da definição de $d_{1}$ que

$$
d_{1}(x)=\lfloor 10 x\rfloor \text { para todo } x \in[0,1)
$$

Vamos ver mais adiante que os outros dígitos $d_{2}(x), d_{3}(x)$ podem ser calculados recursivamente através da equação (2.11), que generaliza a equação (2.9).

Outra função muito útil é a função que dá a parte fracionária de um número $y \in \mathbb{R}$, definida por

$$
\{y\}=y-\lfloor y\rfloor .
$$

Note que todo número real $y$ pode ser decomposto na forma

$$
y=\lfloor y\rfloor+\{y\} .
$$

Lema 2. Para todo $y \in \mathbb{R}$ e $n \in \mathbb{Z},\lfloor y+n\rfloor=\lfloor y\rfloor+n$ e $\{y+n\}=\{y\}$.

Demonstração. Sejam $y \in \mathbb{R}$ e $n \in \mathbb{Z}$. Então

$$
m=\lfloor y+n\rfloor \Longleftrightarrow m \leq y+n<m+1 \Longleftrightarrow m-n \leq y<m-n+1 .
$$


Assim,

$$
\lfloor y\rfloor=m-n=\lfloor y+n\rfloor-n, \quad \text { isto é, } \quad\lfloor y+n\rfloor=\lfloor y\rfloor+n .
$$

Finalmente, observe que

$$
\{y+n\}=y+n-\lfloor y+n\rfloor=y+n-\lfloor y\rfloor-n=y-\lfloor y\rfloor=\{y\} .
$$

Para provar os resultados acerca da expansão decimal de um número, vamos precisar estudar o comportamento da função $f:[0,1) \rightarrow[0,1)$ definida por

$$
f(x)=\{10 x\}, \quad x \in[0,1) .
$$

O gráfico desta função é dado na Figura 2.

Figura 2 - Gráfico da função $f$.

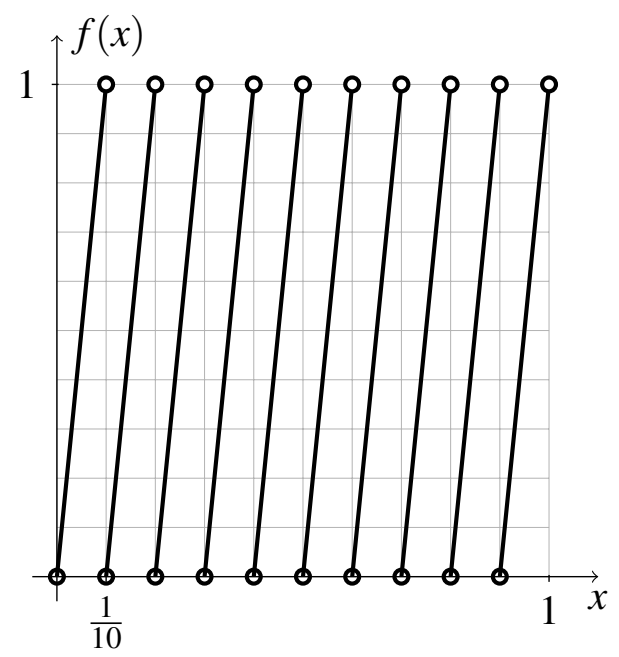

Lema 3. Seja $x \in[0,1)$, então

$$
d_{k}(x)=d_{k-1}(f(x)) \quad \text { para todo } \quad k \geq 2
$$

Demonstração. Seja $x \in[0,1)$ um número tendo expansão decimal

$$
x=0, d_{1} d_{2} \ldots=\sum_{j=1}^{\infty} \frac{d_{j}}{10^{j}} .
$$

Observe que $10 x=d_{1}+y$, onde $y=\sum_{j=1}^{\infty} d_{j+1} 10^{-j}$. Segue da observação 1 que

$$
\sum_{j=1}^{\infty} d_{j+1} 10^{-j}<\sum_{j=1}^{\infty} 9 \cdot 10^{-j}=1, \quad \log 0 \quad 0 \leq y<1
$$


Aplicando o Lema $2 \operatorname{com} n=d_{1}$ resulta

$$
f(x)=\{10 x\}=\left\{y+d_{1}\right\}=\{y\}=y=\sum_{j=1}^{\infty} \frac{d_{j+1}}{10^{j}}=0, d_{2} d_{3} \ldots
$$

Portanto,

$$
d_{k}(x)=d_{k}=d_{k-1}(f(x)) \quad \text { para todo } \quad k \geq 2
$$

Corolário 1. Seja $x \in[0,1)$, então

$$
d_{k}(x)=d_{1}\left(f^{k-1}(x)\right) \quad \text { para todo } \quad k \geq 2
$$

Demonstração. A prova segue por indução sobre $k$. Pelo Lema 3, a afirmação é válida para $k=2$. Suponha agora que a afirmação seja válida para algum $k \geq 2$, isto é, que $\left.d_{k}(y)=d_{1}\left(f^{k-1} y\right)\right)$ para todo $y \in[0,1)$. Segue do Lema 3 e da hipótese de indução que

$$
d_{k+1}(x)=d_{k}(f(x))=d_{1}\left(f^{k-1}(f(x))\right)=d_{1}\left(f^{k}(x)\right) .
$$

Assim, a afirmação é válida para $k+1$. Por indução, a afirmação vale para todo $k \geq 2$.

Teorema 1. Seja $x \in[0,1)$. Considere a sequência $a_{0}, a_{1}, a_{2}, \ldots$ de números do intervalo $[0,1)$ definida recursivamente por

$$
a_{0}=x, \quad a_{k}=f\left(a_{k-1}\right), \quad k \geq 1 .
$$

Então

$$
d_{k}(x)=\left\lfloor 10 a_{k-1}\right\rfloor, \quad k \geq 1 .
$$

Em outras palavras, a expansão decimal de $x$ pode ser calculada através do seguinte algoritmo:

\begin{tabular}{c|ccccc}
$k$ & 0 & 1 & 2 & 3 & $\cdots$ \\
\hline$d_{k}(x)$ & & $\lfloor 10 x\rfloor$ & $\left\lfloor 10 a_{1}\right\rfloor$ & $\left\lfloor 10 a_{2}\right\rfloor$ & $\cdots$ \\
\hline$a_{k}$ & $x$ & $\{10 x\}$ & $\left\{10 a_{1}\right\}$ & $\left\{10 a_{2}\right\}$ & $\cdots$
\end{tabular}

Demonstração. Por definição, $a_{k}=f^{k}(x)$. Assim, $f^{k-1}(x)=a_{k-1}$. Pelo Corolário 1 e pela equação (2.9),

$$
d_{k}(x)=d_{1}\left(f^{k-1}(x)\right)=d_{1}\left(a_{k-1}\right)=\left\lfloor 10 a_{k-1}\right\rfloor .
$$




\subsection{Expansão decimal dos números racionais}

Dizemos que a expansão decimal $0, d_{1} d_{2} \ldots$ de $x \in[0,1)$ é periódica se existirem números inteiros $n_{0}, \tau \geq 1$ tais que $d_{n+\tau}=d_{n}$ para todo $n \geq n_{0}$. Nesse caso, denotamos a expansão decimal de $x$ por

$$
x=0, d_{1} d_{2}, \ldots, d_{n_{0}-1} \overline{d_{n_{0}} d_{n_{0}+1} \ldots d_{n_{0}+\tau-1}} .
$$

Proposição 3. Todo número racional $x \in[0,1)$ admite uma expansão decimal periódica.

Demonstração. Podemos assumir que $x>0, \log x=\frac{p}{q}$, com $p, q \in \mathbb{N}, 1 \leq p<q$ e $p, q$ primos entre si. Pelo Teorema 1 , os dígitos $d_{1}, d_{2}, d_{3}, \ldots$ da expansão decimal de $x$ são definidos por $d_{k}=\left\lfloor 10 a_{k-1}\right\rfloor$, onde a sequência $a_{0}, a_{1}, a_{2}, \ldots$ é definida por:

$$
\begin{aligned}
a_{0} & =x=\frac{p}{q} \\
a_{1} & =f\left(a_{0}\right)=\left\{\frac{10 p}{q}\right\}=\frac{p_{1}}{q} \\
a_{2} & =f\left(a_{1}\right)=\left\{\frac{10 p_{1}}{q}\right\}=\frac{p_{2}}{q} \\
& \vdots \\
a_{k} & =f\left(a_{k-1}\right)=\left\{\frac{10 p_{k-1}}{q}\right\}=\frac{p_{k}}{q}
\end{aligned}
$$

Note que $p_{k} \in\{0,1, \ldots, q-1\}$ para todo $k \geq 0$, onde $p_{0}=p$. Se considerarmos $q+1$ números $p_{0}, p_{1}, \ldots, p_{q}$, pelo menos dois desses números serão iguais. Desta forma, existem $n_{0}, n_{1} \in$ $\{0,1, \ldots, q\}$ tais que $n_{0}<n_{1}$ e $p_{n_{0}}=p_{n_{1}}$. Seja $\tau=n_{1}-n_{0} \in\{1, \ldots, q-1\}$. Afirmamos que

$$
a_{n+\tau}=a_{n} \quad \text { para todo } n \geq n_{0} .
$$

A prova segue por indução sobre $n$. A afirmação vale para $n=n_{0}$ pois

$$
a_{n_{0}+\tau}=\frac{p_{n_{0}+\tau}}{q}=\frac{p_{n_{1}}}{q}=\frac{p_{n_{0}}}{q}=a_{n_{0}} .
$$

Suponha agora que a afirmação seja válida para um certo número $n \geq n_{0}$. Vamos provar que isto implica a validade da afirmação para $n+1$. De fato,

$$
a_{n+1+\tau}=f\left(a_{n+\tau}\right)=f\left(a_{n}\right)=a_{n+1} .
$$

As afirmações (2.11) e (2.12) implicam que

$$
d_{n+\tau}(x)=\left\lfloor 10 a_{n+\tau-1}\right\rfloor=\left\lfloor 10 a_{n-1+\tau}\right\rfloor=\left\lfloor 10 a_{n-1}\right\rfloor=d_{n}(x) \quad \text { para todo } \quad n \geq n_{0}+1 .
$$

Isso mostra que a sequência $d_{1}, d_{2}, \ldots$ é eventualmente periódica. Logo, os números racionais admitem expansão decimal periódica. 
Exemplo 1. A expansão decimal de $\frac{2}{7}$ é $0,285714285714 \ldots=0, \overline{285714}$.

\begin{tabular}{c|cccccccc}
$n$ & 0 & 1 & 2 & 3 & 4 & 5 & 6 & $\cdots$ \\
\hline$d_{n}(x)$ & & 2 & 8 & 5 & 7 & 1 & 4 & $\cdots$ \\
\hline$a_{n}$ & $\frac{2}{7}$ & $\frac{6}{7}$ & $\frac{4}{7}$ & $\frac{5}{7}$ & $\frac{1}{7}$ & $\frac{3}{7}$ & $\frac{2}{7}$ & $\cdots$
\end{tabular}

Exemplo 2. A expansão decimal de $\frac{3}{8}$ é 0,375 .

\begin{tabular}{c|cccccccc}
$n$ & 0 & 1 & 2 & 3 & 4 & 5 & 6 & $\cdots$ \\
\hline$d_{n}(x)$ & & 3 & 7 & 5 & 0 & 0 & 0 & $\cdots$ \\
\hline$a_{n}$ & $\frac{3}{8}$ & $\frac{6}{8}$ & $\frac{4}{8}$ & 0 & 0 & 0 & 0 & $\cdots$
\end{tabular}

Exemplo 3. A expansão decimal de $\frac{2}{9}$ é $0,222 \ldots=0, \overline{2}$.

\begin{tabular}{c|ccccc}
$n$ & 0 & 1 & 2 & 3 & $\cdots$ \\
\hline$d_{n}(x)$ & & 2 & 2 & 2 & $\cdots$ \\
\hline$a_{n}$ & $\frac{2}{9}$ & $\frac{2}{9}$ & $\frac{2}{9}$ & $\frac{2}{9}$ & $\cdots$
\end{tabular}

Proposição 4. Todo número $x \in[0,1)$ que admite expansão decimal periódica é racional.

Demonstração. Seja

$$
x=0, d_{1} d_{2} \ldots d_{n_{0}-1} \overline{d_{n_{0}} d_{n_{0}+1} \ldots d_{n_{0}+\tau-1}}
$$

um número $x$ tendo expansão decimal periódica. Então

$$
10^{n_{0}+\tau-1} x-10^{n_{0}-1} x=d_{1} d_{2} \ldots d_{n_{0}+\tau-1}-d_{1} d_{2} \ldots d_{n_{0}-1}
$$

Portanto,

$$
x=\frac{d_{1} d_{2} \ldots d_{n_{0}+\tau-1}-d_{1} d_{2} \ldots d_{n_{0}-1}}{10^{n_{0}+\tau-1}-10^{n_{0}-1}}
$$

logo $x$ é um número racional.

O seguinte exemplo ajuda a entender a ideia usada na prova da Proposição 4.

Exemplo 4. Seja $x=0.1437 \overline{258}$. Neste caso, temos $n_{0}-1=4$ e $\tau=3$, portanto

$$
x=\frac{1.437 .258-1.437}{10^{7}-10^{4}}=\frac{1.435 .821}{9.990 .000} .
$$


Segue das proposições (3) e (4) a seguinte caracterização dos números racionais.

Teorema 2. Um número $x \in[0,1)$ é racional se e somente se ele admitir expansão decimal periódica.

Um classe especial dos números racionais é formada pelos números cuja expansão decimal termina numa sequência infinita de zeros.

\subsection{Números com expansão decimal finita}

Dizemos que uma expansão decimal $0, d_{1} d_{2} \ldots$ de $x \in[0,1)$ é finita se existir um número natural $n \geq 1$ tal que $d_{k}=0$ para todo $k>n$. Nesse caso, podemos omitir os termos $d_{k} \operatorname{com} k>n$ na equação (2.1), obtendo

$$
x=0, d_{1} d_{2} \ldots d_{n}=\frac{d_{1}}{10}+\frac{d_{2}}{10^{2}}+\cdots+\frac{d_{n}}{10^{n}} .
$$

Teorema 3. Um número $x \in[0,1)$ admite expansão decimal finita se e somente se $x=\frac{p}{10^{m}}$, onde $0 \leq p \leq 10^{m}-1$ e $m \geq 1$.

Demonstração. Se $x=\frac{p}{10^{m}}$, então $x=\sum_{k=1}^{\infty} d_{k} 10^{-k}$, onde $d_{k}=p$ se $k=m$ e $d_{k}=0$ se $k \neq m$. Em particular, $d_{k}=0$ para todo $k \geq m+1$, o que mostra que a expansão de $x$ é finita. Reciprocamente, suponha que $x$ tenha expansão finita, então

$$
x=\frac{d_{1}}{10}+\frac{d_{2}}{10^{2}}+\cdots+\frac{d_{m}}{10^{m}} .
$$

Desta forma, $x=\frac{p}{10^{m}}$, onde $p=10^{m-1} d_{1}+10^{m-2} d_{2}+\cdots+10 d_{m-1}+d_{m}$.

Corolário 2. Um número $x \in(0,1)$ tem expansão decimal finita se e somente se $x=\frac{p}{q}$, onde $p$ e $q$ são relativamente primos e $q$ não tem fatores primos diferentes de 2 e de 5 .

Demonstração. Do Teorema 3, se $x$ tem expansão finita então $x=\frac{p}{10^{m}}=\frac{p}{2^{m} \cdot 5^{m}}$. Logo, cancelando os fatores primos em comum de $p$ e $q=2^{m} \cdot 5^{m}$ resulta numa fração irredutível da forma desejada. Reciprocamente, suponha que $x=\frac{p}{2^{r} 5^{s}}$, onde $r \geq 0$ e $s \geq 0$. Se $r=s$, então $2^{r} \cdot 5^{s}=10^{r}$. Nesse caso, segue do Teorema 3 que $x$ tem expansão finita. Se $r>s$, então $x=\frac{5^{r-s} p}{10^{r}}$ e novamente pelo Teorema 3, $x$ tem expansão finita. O caso $r<s$ é análogo.

Exemplo 5. A expansão de $x=\frac{1}{3}$ é $x=0,333 \ldots$, portanto, ela não é finita. A expansão de $x=\frac{3}{6}$ é finita pois $x=\frac{1}{2}=0,5$. 


\subsection{Unicidade da expansão decimal}

Teorema 4. Todo número $x \in[0,1)$ tem uma única expansão decimal, exceto os números da forma $\frac{p}{10^{m}}$, onde $0 \leq p \leq 10^{m}-1$. Nesse caso, $x$ admite exatamente duas expansões decimais, uma que termina em infinitos zeros consecutivos e outra que termina em infinitos nove consecutivos.

Demonstração. Suponha que $x \in[0,1)$ admita duas expansões decimais:

$$
x=0, a_{1} a_{2} \ldots=0, b_{1} b_{2} \ldots
$$

Seja $a_{k}$ o primeiro dígito em que as duas representações de $x$ diferem. Então $a_{k} \neq b_{k}$, mas

$$
\begin{aligned}
a_{1} & =b_{1} \\
a_{2} & =b_{2} \\
& \vdots \\
a_{k-1} & =b_{k-1} .
\end{aligned}
$$

Vamos supor que $a_{k}<b_{k}$. Como $a_{k}$ e $b_{k}$ são inteiros diferentes, então eles diferem em pelo menos uma unidade, isto é, $b_{k}-a_{k} \geq 1$, ou

$$
a_{k}+1 \leq b_{k}
$$

Considere agora as seguintes desigualdades:

$$
\begin{aligned}
x & =\sum_{1}^{\infty} \frac{a_{n}}{10^{n}} \\
& =\left(\sum_{1}^{k-1} \frac{a_{n}}{10^{n}}\right)+\frac{a_{k}}{10^{k}}+\left(\sum_{k+1}^{\infty} \frac{a_{n}}{10^{n}}\right) \\
& \left.\leq\left(\sum_{1}^{k-1} \frac{a_{n}}{10^{n}}\right)+\frac{a_{k}}{10^{k}}+\left(\sum_{k+1}^{\infty} \frac{9}{10^{n}}\right) \quad \text { (já que } a_{n} \leq 9\right) \\
& =\left(\sum_{1}^{k-1} \frac{a_{n}}{10^{n}}\right)+\frac{a_{k}}{10^{k}}+\left(\frac{9}{10^{k+1}}\right) \text { (soma de progressão geométrica) } \\
& =\left(\sum_{1}^{k-1} \frac{a_{n}}{10^{n}}\right)+\frac{a_{k}}{10^{k}}+\frac{1}{10^{k}} \\
& =\left(\sum_{1}^{k-1} \frac{b_{n}}{10^{n}}\right)+\frac{a_{k}}{10^{k}}+\frac{1}{10^{k}}
\end{aligned}
$$




$$
\begin{aligned}
& =\left(\sum_{1}^{k-1} \frac{b_{n}}{10^{n}}\right)+\frac{a_{k}+1}{10^{k}} \\
& \leq\left(\sum_{1}^{k-1} \frac{b_{n}}{10^{n}}\right)+\frac{b_{k}}{10^{k}} \quad \text { (já que } a_{k}+1 \leq b_{k} \text { pela equação(2.14)) } \\
& \leq\left(\sum_{1}^{k-1} \frac{b_{n}}{10^{n}}\right)+\frac{b_{k}}{10^{k}}+\sum_{k+1}^{\infty} \frac{b_{n}}{10^{n}}=\sum_{1}^{\infty} \frac{b_{n}}{10^{n}}=x
\end{aligned}
$$

Temos uma lista de expressões entre $x$ e $x$, então todas as expressões acima precisam ser iguais. Em particular,

$$
\left(\sum_{1}^{k-1} \frac{b_{n}}{10^{n}}\right)+\frac{b_{k}}{10^{k}}=\left(\sum_{1}^{k-1} \frac{b_{n}}{10^{n}}\right)+\frac{b_{k}}{10^{k}}+\sum_{k+1}^{\infty} \frac{b_{n}}{10^{n}} .
$$

Portanto,

$$
\sum_{k+1}^{\infty} \frac{b_{n}}{10^{n}}=0
$$

Na equação (2.15) temos uma sequência de números não-negativos cuja soma é zero. A única maneira que isso pode acontecer é se todos os b's em (2.15) forem 0. Isto é, se $b_{k+1}=b_{k+2}=b_{k+3}=\cdots=0$. Isto quer dizer que $x$ admite uma expansão decimal finita. Pelo Teorema 3, $x=\frac{p}{10^{m}}$ para algum $0 \leq p \leq 10^{m}-1$ e $m \geq 1$.

Reciprocamente, se $x=\frac{p}{10^{m}}$ para algum $0 \leq p \leq 10^{m}-1$ e $m \geq 1$, então pelo Teorema 3 $x$ admite uma expansão finita da forma:

$$
x=0, a_{1} a_{2} \ldots a_{k-1} a_{k} .
$$

Afirmamos que

$$
0, a_{1} a_{2} \ldots a_{k-1}\left(a_{k}-1\right) 999 \ldots
$$

também é uma expansão decimal de $x$. De fato,

$$
\begin{aligned}
0, a_{1} a_{2} \ldots a_{k-1}\left(a_{k}-1\right) 999 \ldots & =\sum_{n=1}^{k-1} \frac{a_{n}}{10^{n}}+\frac{a_{k}-1}{10^{k}}+\sum_{n=k+1}^{\infty} \frac{9}{10^{n}} \\
& =\sum_{n=1}^{k-1} \frac{a_{n}}{10^{n}}+\frac{a_{k}-1}{10^{k}}+\frac{\frac{9}{10^{k+1}}}{1-\frac{1}{10}} \\
& =\sum_{n=1}^{k-1} \frac{a_{n}}{10^{n}}+\frac{a_{k}-1}{10^{k}}+\frac{1}{10^{k}} \\
& =\sum_{n=1}^{k} \frac{a_{n}}{10^{n}}=x .
\end{aligned}
$$




\subsection{Expansão decimal de números irracionais quadráticos}

Os números irracionais mais simples são os números irracionais quadráticos. Um número irracional $x \in[0,1)$ é um irracional quadrático se ele for raiz de uma equação quadrática da forma $a^{2} x+b x+c=0$, onde $a, b$ e $c$ são números inteiros com $a \neq 0$. Assim, são números da forma:

$$
\frac{-b \pm \sqrt{b^{2}-4 a c}}{2 a}
$$

Um exemplo típico de um número irracional quadrático são as raízes quadradas $\sqrt{p}$, onde $p$ é um número natural que não é quadrado perfeito. Isto é ilustrado pelo seguinte resultado.

Teorema 5. Se $p$ é um número natural que não é quadrado perfeito, então $\sqrt{p}$ é irracional.

Demonstração. Suponha que $\sqrt{p}$ seja racional, então existem inteiros positivos $a, b$ relativamente primos tais que $\sqrt{p}=\frac{a}{b}$, isto é, $a^{2}=b^{2} p$. Isto mostra que o número $b^{2} p$ é produto de um número par de números primos, portanto $p$ é produto de um número par de números primos. Logo, $p$ é um quadrado perfeito.

Na próxima seção vamos estudar o Método Babilônico para gerar uma sequência de aproximações racionais de um número irracional quadrático.

\subsection{O Método Babilônico para encontrar $\sqrt{p}$}

O método babilônico para encontrar $\sqrt{p}$ consiste em gerar uma sequência $x_{0}, x_{1}, x_{2}, \ldots$ de números racionais que converge para $\sqrt{p}$.

Teorema 6 (Método babilônico). A sequência $x_{0}, x_{1}, x_{2}, \ldots$ definida recursivamente por

$$
x_{0}=p, \quad x_{n+1}=\frac{1}{2}\left(x_{n}+\frac{p}{x_{n}}\right), \quad n \geq 0,
$$

é convergente e $\lim _{n \rightarrow \infty} x_{n}=\sqrt{p}$. Além disso, o erro de aproximação no enésimo iterado é dado por:

$$
\left|x_{n}-\sqrt{p}\right| \leq\left(\frac{1}{2}\right)^{n}|p-\sqrt{p}|, \quad n \geq 1 .
$$

Demonstração. A prova segue das diversas afirmações dadas abaixo.

Afirmação 1.

$$
x_{n}>0 \quad \text { para todo } n \geq 0 \text {. }
$$

De fato, isto segue facilmente de (2.17) por indução sobre $n$. 
Afirmação 2.

$$
\sqrt{p} \leq x_{n} \leq p \quad \text { para todo } n \geq 0
$$

A Afirmação 2 vale para $n=0$ pois $x_{0}=p$. Suponha que a afirmação seja válida para algum $n \geq 0$ (hipótese de indução). Então de (2.17) segue que

$$
\begin{aligned}
x_{n+1}^{2} & =\frac{1}{4}\left(x_{n}+\frac{p}{x_{n}}\right)^{2}=\frac{1}{4}\left(x_{n}^{2}+2 p+\frac{p^{2}}{x_{n}^{2}}\right)=\frac{1}{4}\left(x_{n}^{2}-2 p+\frac{p^{2}}{x_{n}^{2}}\right)+p \\
& =\frac{1}{4}\left(x_{n}-\frac{p}{x_{n}}\right)^{2}+p \geq p .
\end{aligned}
$$

Assim, $x_{n+1}^{2} \geq p$. Da Afirmação 1 conclui-se que

$$
x_{n+1} \geq \sqrt{p}
$$

Da hipótese de indução e de (2.17) também segue que

$$
x_{n+1}=\frac{x_{n}}{2}+\frac{p}{2 x_{n}} \leq \frac{p}{2}+\frac{p}{2 \sqrt{p}}=\frac{p}{2}+\frac{\sqrt{p}}{2} \leq p .
$$

De (2.19) e (2.20) segue que

$$
\sqrt{p} \leq x_{n+1} \leq p
$$

Assim, por indução sobre $n$ conclui-se que a Afirmação 2 vale para todo $n \geq 0$.

Afirmação 3.

$$
\left|1-\frac{\sqrt{p}}{x_{n}}\right| \leq 1 \quad \text { para todo } \quad n \geq 0 .
$$

De fato, as seguintes desigualdades seguem da Afirmação 2.

$$
\begin{aligned}
\frac{1}{p} \leq \frac{1}{x_{n}} & \leq \frac{1}{\sqrt{p}} . \\
\frac{1}{\sqrt{p}} \leq \frac{\sqrt{p}}{x_{n}} & \leq 1 . \\
-1 \leq-\frac{\sqrt{p}}{x_{n}} & \leq-\frac{1}{\sqrt{p}} . \\
0 \leq 1-\frac{\sqrt{p}}{x_{n}} & \leq 1-\frac{1}{\sqrt{p}}<1 .
\end{aligned}
$$

Assim,

$$
\left|1-\frac{\sqrt{p}}{x_{n}}\right|=1-\frac{\sqrt{p}}{x_{n}} \leq 1
$$

Isto prova a Afirmação 3. 
Afirmação 4.

$$
\left|x_{n+1}-\sqrt{p}\right| \leq \frac{1}{2}\left|x_{n}-\sqrt{p}\right| \text { para todo } \quad n \geq 0
$$

De fato, segue de (2.17) que para todo $n \geq 0$,

$$
x_{n+1}-\sqrt{p}=\frac{1}{2}\left(x_{n}-\sqrt{p}+\frac{p}{x_{n}}-\sqrt{p}\right)=\frac{1}{2}\left(1-\frac{\sqrt{p}}{x_{n}}\right)\left(x_{n}-\sqrt{p}\right)
$$

Passando o valor absoluto na desigualdade (2.21) e usando a Afirmação 3, resulta

$$
\left|x_{n+1}-\sqrt{p}\right| \leq \frac{1}{2}\left|x_{n}-\sqrt{p}\right|,
$$

o que prova a Afirmação 4.

Para concluir a demonstração, precisamos provar a desigualdade (2.18). A desigualdade vale para $n=0$. Suponha que ela seja válida para algum $n \geq 0$ (hipótese de indução). Então da Afirmação 4 segue que

$$
\left|x_{n+2}-\sqrt{p}\right| \leq \frac{1}{2}\left|x_{n+1}-\sqrt{p}\right| \leq \frac{1}{2} \cdot\left(\frac{1}{2}\right)^{n}|p-\sqrt{p}|=\left(\frac{1}{2}\right)^{n+1}|p-\sqrt{p}| .
$$

Isto é, (2.18) vale para $n+1$ e por indução segue que ela vale para todo $n \geq 0$.

Exemplo 6. Encontre $\sqrt{2}$ com 5 casas decimais de precisão.

Resolução. Para obter 5 casas decimais de precisão, devemos ter $\left|x_{n}-\sqrt{2}\right|<10^{-6}$. De (2.18), uma desigualdade deste tipo será conseguida se impormos que $\left(\frac{1}{2}\right)^{n} 2<10^{-6}$, pois $2-\sqrt{2}<2$. Aplicando logaritmo na desigualdade anterior resulta que $-(n-1) \log 2<-6$, isto é, $n>6 / \log 2+1$. Assim, $n=21$ iterações assegura que o erro é menor que $10^{-6}$.

Na Tabela 1 foram impressas as primeiras 21 iterações do Método Babilônico para encontrar $\sqrt{2}$. Pela justificativa dada acima, temos que $\sqrt{2}$ com as 5 casas decimais de precisão é dado por

$$
\sqrt{2}=1,41421
$$

Exemplo 7. Encontre $\sqrt{3}$ com 5 casas decimais de precisão.

Resolução. Para obter 5 casas decimais de precisão, devemos ter $\left|x_{n}-\sqrt{3}\right|<10^{-6}$. De (2.18), uma desigualdade deste tipo será conseguida se impormos que $\left(\frac{1}{2}\right)^{n} 3<10^{-6}$, pois $3-\sqrt{3}<3$. Aplicando logaritmo na desigualdade anterior resulta que $-n \log 2+\log 3<-6$, isto é, $n>(6+\log 3) / \log 2$. Assim, $n=22$ iterações assegura que o erro é menor que $10^{-6}$. 
Tabela 1 - Tabela com as primeiras 21 iterações do Método Babilônico para $\sqrt{2}$.

\begin{tabular}{c|c}
$n$ & $x_{n}$ \\
\hline 0 & 2.000000000000000000000000000000000000000 \\
1 & 1.500000000000000000000000000000000000000 \\
2 & 1.416666666666666666666666666666666666667 \\
3 & 1.414215686274509803921568627450980392157 \\
4 & 1.414213562374689910626295578890134910117 \\
5 & 1.414213562373095048801689623502530243615 \\
6 & 1.414213562373095048801688724209698078570 \\
7 & 1.414213562373095048801688724209698078570 \\
8 & 1.414213562373095048801688724209698078570 \\
9 & 1.414213562373095048801688724209698078570 \\
10 & 1.414213562373095048801688724209698078570 \\
11 & 1.414213562373095048801688724209698078570 \\
12 & 1.414213562373095048801688724209698078570 \\
13 & 1.414213562373095048801688724209698078570 \\
14 & 1.414213562373095048801688724209698078570 \\
15 & 1.414213562373095048801688724209698078570 \\
16 & 1.414213562373095048801688724209698078570 \\
17 & 1.414213562373095048801688724209698078570 \\
18 & 1.414213562373095048801688724209698078570 \\
19 & 1.414213562373095048801688724209698078570 \\
20 & 1.414213562373095048801688724209698078570 \\
21 & 1.414213562373095048801688724209698078570
\end{tabular}

$\mathrm{Na}$ Tabela 2 foram impressas as primeiras 22 iterações do Método Babilônico para encontrar $\sqrt{3}$. Pela justificativa dada acima, temos que $\sqrt{3}$ com as 5 casas decimais de precisão é dado por

$$
\sqrt{3}=1,73205
$$

Exemplo 8. Encontre $\sqrt{17}$ com 4 casas decimais de precisão.

Resolução. Para obter 4 casas decimais de precisão, devemos ter $\left|x_{n}-\sqrt{17}\right|<10^{-5}$. De (2.18), uma desigualdade deste tipo será conseguida se impormos que $\left(\frac{1}{2}\right)^{n} 17<10^{-5}$, pois $17-\sqrt{17}<17$. Aplicando logaritmo na desigualdade anterior resulta que $-n \log 2+\log 17<-5$, isto é, $n>(5+\log 17) / \log 2$. Assim, $n=21$ iterações assegura que o erro é menor que $10^{-5}$.

Na Tabela 3 foram impressas as primeiras 21 iterações do Método Babilônico para encontrar $\sqrt{17}$. Pela justificativa dada acima, temos que $\sqrt{17}$ com as 4 casas decimais de precisão é dado por

$$
\sqrt{17}=4,1231
$$


Tabela 2 - Tabela com as primeiras 22 iterações do Método Babilônico para $\sqrt{3}$.

\begin{tabular}{c|c}
$n$ & $x_{n}$ \\
\hline 0 & 3.000000000000000000000000000000000000000 \\
1 & 2.000000000000000000000000000000000000000 \\
2 & 1.750000000000000000000000000000000000000 \\
3 & 1.732142857142857142857142857142857142857 \\
4 & 1.732050810014727540500736377025036818851 \\
5 & 1.732050807568877295254353946072171914235 \\
6 & 1.732050807568877293527446341505872367804 \\
7 & 1.732050807568877293527446341505872366943 \\
8 & 1.732050807568877293527446341505872366943 \\
9 & 1.732050807568877293527446341505872366943 \\
10 & 1.732050807568877293527446341505872366943 \\
11 & 1.732050807568877293527446341505872366943 \\
12 & 1.732050807568877293527446341505872366943 \\
13 & 1.732050807568877293527446341505872366943 \\
14 & 1.732050807568877293527446341505872366943 \\
15 & 1.732050807568877293527446341505872366943 \\
16 & 1.732050807568877293527446341505872366943 \\
17 & 1.732050807568877293527446341505872366943 \\
18 & 1.732050807568877293527446341505872366943 \\
19 & 1.732050807568877293527446341505872366943 \\
20 & 1.732050807568877293527446341505872366943 \\
21 & 1.732050807568877293527446341505872366943 \\
22 & 1.732050807568877293527446341505872366943
\end{tabular}

\subsubsection{Outra prova de que $\lim _{n \rightarrow \infty} x_{n}=\sqrt{p}$}

Observa-se nas três tabelas anteriores que a sequência $\left(x_{n}\right)$ é decrescente. Isto não é fortuito. De fato, segue da Afirmação 2 no Teorema 6 e da equação (2.17) que

$$
\frac{x_{n+1}}{x_{n}}=\frac{1}{2}\left(1+\frac{p}{x_{n}^{2}}\right) \leq \frac{1}{2}\left(1+\frac{p}{(\sqrt{p})^{2}}\right)=1 .
$$

Isto é,

$$
x_{n+1} \leq x_{n} \quad \text { para todo } \quad n \geq 0 .
$$

Desta forma, a sequência $\left(x_{n}\right)$ é decrescente e limitada inferiormente por $\sqrt{p}$. Pelo item (b) do Lema 1 , existe $x \in \mathbb{R}$ tal que $\lim _{n \rightarrow \infty} x_{n}=x$. Afirmamos que $x=\sqrt{p}$. De fato, $\lim _{n \rightarrow \infty} x_{n}=x=$ $\lim _{n \rightarrow \infty} x_{n+1}$. Passando o limite em (2.17), obtemos:

$$
x=\frac{1}{2}\left(x+\frac{p}{x}\right) .
$$

Assim, multiplicando por $x$, resulta que

$$
2 x^{2}=x^{2}+p, \quad \text { isto é, } \quad x=-\sqrt{p} \quad \text { ou } \quad x=\sqrt{p} .
$$

De (2.17) segue que $x \geq \sqrt{p}$, portanto $\lim _{n \rightarrow \infty} x_{n}=x=\sqrt{p}$. 
Tabela 3 - Tabela com as primeiras 21 iterações do Método Babilônico para $\sqrt{17}$.

\begin{tabular}{c|c}
$n$ & $x_{n}$ \\
\hline 0 & 17.00000000000000000000000000000000000000 \\
1 & 9.000000000000000000000000000000000000000 \\
2 & 5.444444444444444444444444444444444444444 \\
3 & 4.283446712018140589569160997732426303855 \\
4 & 4.126106627581330750051917714324127392266 \\
5 & 4.123106716962795179994990777747392253942 \\
6 & 4.123105625617804983895597281255081612160 \\
7 & 4.123105625617660549821409858503869332281 \\
8 & 4.123105625617660549821409855974077025147 \\
9 & 4.123105625617660549821409855974077025147 \\
10 & 4.123105625617660549821409855974077025147 \\
11 & 4.123105625617660549821409855974077025147 \\
12 & 4.123105625617660549821409855974077025147 \\
13 & 4.123105625617660549821409855974077025147 \\
14 & 4.123105625617660549821409855974077025147 \\
15 & 4.123105625617660549821409855974077025147 \\
16 & 4.123105625617660549821409855974077025147 \\
17 & 4.123105625617660549821409855974077025147 \\
18 & 4.123105625617660549821409855974077025147 \\
19 & 4.123105625617660549821409855974077025147 \\
20 & 4.123105625617660549821409855974077025147 \\
21 & 4.123105625617660549821409855974077025147 \\
22 & 4.123105625617660549821409855974077025147
\end{tabular}

\subsection{Outras abordagens do Método Babilônico}

Encontrar o valor numérico de $\sqrt{p}$ é equivalente a encontrar a solução positiva da equação $f(x)=0$, onde $f(x)=x^{2}-p$. Isto é, $x^{*}=\sqrt{p}$ se e somente se $f\left(x^{*}\right)=0$ e $x^{*}>0$. Problemas desta natureza são conhecidos como o problema de encontrar o zero de uma equação polinomial. Uma outra categoria de problemas consiste em se encontrar o ponto fixo de uma função $g$, isto é, achar um ponto $x^{*}$ tal que $g\left(x^{*}\right)=x^{*}$. Um problema do primeiro tipo sempre pode ser transformado num problema do segundo tipo. Há diversas maneiras de escolher uma função $g$ que realiza esta transformação. Um método conveniente de achar um candidato a função $g$ é conhecido como Método de Newton. Ele consiste em definir $g$ da seguinte maneira:

$$
g(x)=x-\frac{f(x)}{f^{\prime}(x)}
$$

Para aplicar o Método de Newton supõe-se que $f$ é continuamente diferenciável e não se anula numa vizinhança do ponto fixo $x^{*}$. Isto é equivalente a requerer que $f^{\prime}\left(x^{*}\right) \neq 0$. Observe que $x^{*}$ é zero de $f$ se e somente se $x^{*}$ é ponto fixo da $g$ definida por (2.22).

O método clássico de se encontrar o ponto fixo de uma função $g$ consiste no seguinte 
procedimento. Escolhe-se $x_{0}$ suficientemente próximo do ponto fixo $x^{*}$ cujo valor numérico se deseja determinar. Então define-se a sequência $x_{1}, x_{2}, \ldots$ pela lei de recorrência $x_{n+1}=g\left(x_{n}\right)$ para todo $n \geq 0$. Pode-se provar que sob condições adequadas sobre a função $g$ (isto é, se $g$ for uma contração), a sequência $\left(x_{n}\right)$ converge para o ponto fixo $x^{*}$ de $g$. No caso em que se deseja determinar $\sqrt{p}$ temos que $f(x)=x^{2}-p$. Sendo assim,

$$
g(x)=x-\frac{f(x)}{f^{\prime}(x)}=x-\frac{x^{2}-p}{2 x}=\frac{2 x^{2}-x^{2}+p}{2 x}=\frac{1}{2}\left(x+\frac{p}{x}\right) .
$$

e

$$
x_{n+1}=g\left(x_{n}\right)=\frac{1}{2}\left(x_{n}+\frac{p}{x_{n}}\right), \quad n \geq 0 .
$$

Note que escolhendo $x_{n}=p$ resulta em uma sequência idêntica àquela definida por (2.23). Abaixo apresentamos uma abordagem desta sequência usando o Teorema do Ponto Fixo de Banach. Vamos precisar de alguns conceitos e resultados. Vamos assumir que $a<b$ são dois números reais e que $[a, b]$ é o intervalo compacto com extremos em $a$ e $b$.

Definição 1. Um ponto $x^{*} \in[a, b]$ é considerado ponto fixo de uma função $g:[a, b] \rightarrow \mathbb{R}$ se $g\left(x^{*}\right)=x^{*}$.

Exemplo 9. Os pontos fixos da função $g(x)=x^{3}-x^{2}+1$ são $x_{1}^{*}=1$ e $x_{2}^{*}=-1$.

Resolução.

$$
\begin{aligned}
g(x) & =x \\
x^{3}-x^{2}+1 & =x \\
x^{3}-x^{2}-x+1 & =0 \\
x^{2}(x-1)-(x-1) & =0 \\
\left(x^{2}-1\right)(x-1) & =0 \\
(x-1)(x+1)(x-1) & =0 \\
(x-1)^{2}(x+1) & =0 \\
x=1 \text { ou } x & =-1
\end{aligned}
$$

Logo, os pontos fixos são $x_{1}^{*}=1$ e $x_{2}^{*}=-1$.

Teorema 7. Seja $f:[a, b] \rightarrow \mathbb{R}$ uma função contínua. Se $f(a) \cdot f(b) \leq 0$ então f tem um zero em $[a, b]$.

Demonstração. Se $f(a)=0$ ou $f(b)=0$, então f tem um zero em $[a, b]$. Se $f(a) \neq 0$ e $f(b) \neq 0$ então $f(a) \cdot f(b)<0$. Assim, o intervalo com extremos em $f(a)$ e $f(b)$ contém o zero. Pelo Teorema do Valor Intermediário, existe $x^{*} \in(a, b)$ tal que $f\left(x^{*}\right)=0$. 
Teorema 8 (Teorema do Ponto Fixo de Brouwer). Seja $g:[a, b] \rightarrow \mathbb{R}$ uma função contínua tal que $g(x) \in[a, b]$ para todo $x \in[a, b]$, isto é, $g$ leva $[a, b]$ em $[a, b]$. Então $g$ tem pelo menos um ponto fixo em $[a, b]$.

Demonstração. Seja $f(x)=x-g(x)$. Então o fato de que $a \leq g(a) \leq b$ implica que $f(a)=$ $a-g(a) \leq 0$ e o fato de que $a \leq g(b) \leq b$ implica que $f(b)=b-g(b) \geq 0$. Assim, pelo teorema 7 , exite $x^{*} \in[a, b]$ tal que $f\left(x^{*}\right)=0, \log$ o, $x^{*}-g\left(x^{*}\right)=0$, portanto $g\left(x^{*}\right)=x^{*}$.

Exemplo 10. A função $g:[1,2] \rightarrow \mathbb{R}$ definida por $g(x)=\ln (2 x+1)$ tem ponto fixo.

Resolução. $g(1)=\ln 3 \in[1,2]$ e $g(2)=\ln 5 \in[1,2]$. A função $g$ é crescente, portanto

$$
1 \leq g(1) \leq g(x) \leq g(2) \leq 2 \quad \text { para todo } \quad x \in[1,2]
$$

Desta forma, $g(x) \in[1,2]$ para todo $x \in[1,2]$. Além disso, $g$ é contínua no intervalo [1,2]. Pelo Teorema do Ponto Fixo de Brouwer, $g$ tem um ponto fixo em $[1,2]$.

Definição 2. Uma função $g:[a, b] \rightarrow \mathbb{R}$ é uma contração se existir uma constante $0 \leq L<1$ tal que

$$
|g(x)-g(y)| \leq L|x-y| \text { para todo } x, y \in[a, b] .
$$

Neste caso, dizemos que $g$ é uma L-contração.

Teorema 9 (Teorema do Ponto Fixo de Banach). Seja $g:[a, b] \rightarrow \mathbb{R}$ uma contração tal que $g(x) \in[a, b]$ para todo $x \in[a, b]$, isto é, $g$ leva $[a, b]$ em $[a, b]$. Então $g$ tem um único ponto fixo. Além disso, para todo $x_{0} \in[a, b]$, a sequência $x_{0}, x_{1}, x_{2}, \ldots$ definida por $x_{n+1}=g\left(x_{n}\right)$ converge para o único ponto fixo de $g$.

Demonstração. A existência de pelo menos um ponto fixo é garantida pelo Teorema do Ponto Fixo de Brouwer. A unicidade é provada da seguinte maneira. Sejam $x^{*}, y^{*}$ pontos fixos de $g$. Então:

$$
\left|x^{*}-y^{*}\right|=\left|g\left(x^{*}\right)-g\left(y^{*}\right)\right| \leq L\left|x^{*}-y^{*}\right|
$$

Portanto,

$$
\begin{array}{r}
\left|x^{*}-y^{*}\right| \leq L\left|x^{*}-y^{*}\right| \\
\left|x^{*}-y^{*}\right|-L\left|x^{*}-y^{*}\right| \leq 0 \\
0 \leq(1-L)\left|x^{*}-y^{*}\right| \leq 0
\end{array}
$$

Logo, $(1-L)\left|x^{*}-y^{*}\right|=0$ e, portanto $x^{*}=y^{*}$ pois $L<1$. Observe que:

$$
\left|x_{n}-x^{*}\right|=\left|g\left(x_{n-1}\right)-g\left(x^{*}\right)\right| \leq L\left|x_{n-1}-x^{*}\right| \leq \cdots \leq L^{n}\left|x_{0}-x^{*}\right| .
$$

Como $L^{n} \rightarrow 0$ quando $n \rightarrow \infty$, temos que $\lim _{n \rightarrow \infty}\left|x_{n}-x^{*}\right|=0$. 
Exemplo 11. Mostre que a função $g:[1,2] \rightarrow \mathbb{R}$ definida por $g(x)=\frac{1}{2}\left(x+\frac{2}{x}\right)$ satisfaz todas as hipóteses do Teorema do Ponto Fixo de Banach. Conclua que a sequência $x_{0}, x_{1}, x_{2}, \ldots$ definida recursivamente por $x_{n+1}=g\left(x_{n}\right)$ converge para $\sqrt{2}$ para todo $x_{0} \in[1,2]$.

Resolução. O primeiro passo é mostrar que $g$ leva o intervalo $[1,2]$ nele mesmo, isto é, que $g([1,2]) \subset[1,2]$. Isto é consequência das seguintes desigualdades:

$$
1 \leq x \leq 2 \Rightarrow 1 \leq \frac{2}{x} \leq 2 \Rightarrow 2 \leq x+1 \leq x+\frac{2}{x} \leq x+2 \leq 4 \Rightarrow 1 \leq \frac{1}{2}\left(x+\frac{2}{x}\right) \leq 2 .
$$

Assim, $g$ leva o intervalo $[1,2]$ nele mesmo.

Para mostrar que $g$ é uma contração, vamos precisar provar a seguinte desigualdade:

$$
\left|1-\frac{2}{x y}\right| \leq 1
$$

A desigualdade (2.24) segue das seguintes desigualdades:

$$
1 \leq x \leq 2,1 \leq y \leq 2 \Rightarrow 1 \leq x y \leq 4 \Rightarrow \frac{1}{4} \leq \frac{1}{x y} \leq 1 \Rightarrow \frac{1}{2} \leq \frac{2}{x y} \leq 2 .
$$

Portanto,

$$
-1 \leq 1-\frac{2}{x y} \leq \frac{1}{2} \leq 1 \quad \text { e } \quad\left|1-\frac{2}{x y}\right| \leq 1 .
$$

Vamos mostrar agora que $g$ é uma $\frac{1}{2}$-contração. Segue da definição de $g$ e de (2.24) que:

$$
\begin{aligned}
|g(x)-g(y)| & =\left|\frac{1}{2}\left(x+\frac{p}{x}\right)-\frac{1}{2}\left(y+\frac{p}{y}\right)\right| \\
& =\left|\frac{1}{2}\left(x-y+\frac{p}{x}-\frac{p}{y}\right)\right| \\
& =\left|\frac{1}{2}\left(x-y-\frac{p(x-y)}{x y}\right)\right| \\
& =\left|\frac{1}{2}(x-y)\left(1-\frac{p}{x y}\right)\right| \\
& \leq \frac{1}{2}\left|1-\frac{p}{x y}\right| \cdot|x-y| \\
& \leq \frac{1}{2}|x-y| .
\end{aligned}
$$

Isto mostra que $g$ é contração em $[1,2]$. Além disso, $g(\sqrt{2})=\sqrt{2}$, isto é, $\sqrt{2}$ é ponto fixo de $g$. Pelo Teorema do Ponto Fixo de Banach, a sequência $x_{0}, x_{1}, x_{2}, \ldots$ definida recursivamente por $x_{n+1}=g\left(x_{n}\right)$ converge para $\sqrt{2}$ independentemente da escolha inicial de $x_{0} \in[1,2]$.

Vamos abordar agora o problema da precisão na aproximação do ponto fixo. Por exemplo, já vimos que se $g(x)=\frac{1}{2}\left(x+\frac{2}{x}\right)$ então $\lim _{n \rightarrow \infty} g^{n}(1)=\sqrt{2}$. Note que $g^{n}(1)$ é um número racional que aproxima o valor de $\sqrt{2}$. Queremos estimar o erro cometido na aproximação. Uma estimativa da velocidade de convergência é dada no próximo teorema. 
Teorema 10. Seja $g:[a, b] \rightarrow[a, b]$ uma L-contração. Dados $x_{0} \in[a, b]$ e $\varepsilon>0$, seja

$$
N=\left\lfloor\frac{\ln \left|g\left(x_{0}\right)-x_{0}\right|-\ln (\varepsilon(1-L))}{\ln \left(\frac{1}{L}\right)}\right\rfloor+1 .
$$

Então $\left|x_{n}-x^{*}\right| \leq \varepsilon$ para todo $n \geq N$, onde $x^{*}$ é o único ponto fixo de $g$ e $x_{n}=g^{n}\left(x_{0}\right)$.

Demonstração. Na demonstração do Teorema do Ponto Fixo de Banach, a seguinte desigualdade foi obtida:

$$
\left|x_{n}-x^{*}\right| \leq L^{n}\left|x_{0}-x^{*}\right| \text { para todo } n \geq 1
$$

Para $n=1$, temos $\left|x_{1}-x^{*}\right| \leq L\left|x_{0}-x^{*}\right|, \log$ :

$$
\left|x_{0}-x^{*}\right|=\left|x_{0}-x_{1}+x_{1}-x^{*}\right| \leq\left|x_{0}-x_{1}\right|+\left|x_{1}-x^{*}\right| \leq\left|x_{0}-x_{1}\right|+L\left|x_{0}-x^{*}\right| .
$$

Assim,

$$
\left|x_{0}-x^{*}\right| \leq \frac{1}{1-L}\left|x_{0}-x_{1}\right|
$$

Substituindo em (2.25), obtemos:

$$
\left|x_{n}-x^{*}\right| \leq \frac{L^{n}}{1-L}\left|x_{0}-x_{1}\right|
$$

Portanto, a condição $\left|x_{n}-x^{*}\right| \leq \varepsilon$ será satisfeita se

$$
L^{n} \frac{1}{1-L}\left|x_{1}-x_{0}\right| \leq \varepsilon
$$

Aplicando ln em (2.26), resulta nas seguintes desigualdades:

$$
\begin{gathered}
\frac{L^{n}}{1-L}\left|x_{1}-x_{0}\right| \leq \varepsilon \\
L^{n}\left|x_{1}-x_{0}\right| \leq \varepsilon(1-L) \\
\ln \left(L^{n}\left|x_{1}-x_{0}\right|\right) \leq \ln (\varepsilon(1-L)) \\
\ln \left(L^{n}\right)+\ln \left|x_{1}-x_{0}\right| \leq \ln (\varepsilon(1-L)) \\
n \ln L+\ln \left|x_{1}-x_{0}\right| \leq \ln (\varepsilon(1-L)) \\
\ln \left|x_{1}-x_{0}\right|-\ln (\varepsilon(1-L)) \leq-n \ln L=n \ln \left(\frac{1}{L}\right) \\
\frac{\ln \left|g\left(x_{0}\right)-x_{0}\right|-\ln (\varepsilon(1-L))}{\ln \left(\frac{1}{L}\right)} \leq n .
\end{gathered}
$$

Observação 2. No Teorema 10, se quisermos que a aproximação $x_{n}$ do ponto fixo $x^{*}$ tenha $r$ casas decimais de precisão então basta tomar $\varepsilon<10^{-(r+1)}$.

Exemplo 12. Encontrar a expansão decimal de $\sqrt{2}$ com 4 casas decimais de precisão. 
Resolução. Já vimos que $\sqrt{2}$ é o único ponto fixo da L-contração $g(x)=\frac{1}{2}\left(x+\frac{2}{x}\right)$, onde $L=\frac{1}{2}$. Tomando $\varepsilon=10^{-5}, x_{0}=1$ e calculando o valor de $\mathrm{N}$ no teorema 10 , obtemos:

$$
N=\frac{\ln |g(1)-1|-\ln \left(10^{-5}\left(1-\frac{1}{2}\right)\right)}{\ln \left(\frac{1}{\frac{1}{2}}\right)}=\lfloor 16.6096\rfloor+1=16+1=17 .
$$

Portanto, $\sqrt{2}=1,4142$ pois $x_{17}=1.4142135623730950 \ldots$ 
CAPÍTULO

\section{3}

\section{REGULARIDADE DOS DÍGITOS DA EXPANSÃO DECIMAL}

\subsection{Conjuntos de medida zero e de medida total}

Dizemos que um conjunto $S \subset[0,1)$ tem medida zero se para todo $\varepsilon>0$ existir uma família enumerável de intervalos abertos $J_{1}, J_{2}, \ldots$ tais que $S \subset \bigcup_{n=1}^{\infty} J_{n}$ e $\sum_{n=1}^{\infty}\left|J_{n}\right|<\varepsilon$, onde $\left|J_{n}\right|$ denota o comprimento do intervalo $J_{n}$. Um conjunto $S \subset[0,1)$ tem medida total se o complemento $[0,1) \backslash S$ tiver medida zero.

Lema 4. Todo subconjunto enumerável $S=\left\{x_{1}, x_{2}, \ldots\right\}$ de $[0,1)$ tem medida zero.

Demonstração. Seja $\varepsilon>0$ dado. Para cada $n \geq 1$, seja $J_{n}=\left(x_{n}-\frac{\varepsilon}{2^{n+1}}, x_{n}+\frac{\varepsilon}{2^{n+1}}\right)$. Então $S \subset \bigcup_{n=1}^{\infty} J_{n},\left|J_{n}\right|=\frac{\varepsilon}{2^{n}} \mathrm{e}$

$$
\sum_{n=1}^{\infty}\left|J_{n}\right|=\sum_{n=1}^{\infty} \frac{\varepsilon}{2^{n}}=\varepsilon
$$

Corolário 3. O conjunto $\mathbb{Q} \cap[0,1)$ dos números racionais do intervalo $[0,1)$ tem medida zero. O conjunto $[0,1) \backslash \mathbb{Q}$ dos números irracionais do intervalo $[0,1)$ tem medida total.

Demonstração. Segue do fato de $\mathbb{Q}$ ser um conjunto enumerável.

\subsection{Números simplesmente normais}

Dizemos que um número $x \in[0,1)$ é simplesmente normal se cada dígito $d \in\{0,1, \ldots, 9\}$ aparece com frequência de $10 \%$ na expansão decimal de $x$. Mais especificamente, seja $x \in[0,1)$ 
um número tendo expansão decimal $x=0, d_{1} d_{2}, \ldots$ Para cada $d \in\{0,1, \ldots, 9\}$ defina

$$
\mathbb{P}(d)=\lim _{n \rightarrow \infty} \frac{\#\left\{1 \leq k \leq n \mid d_{k}=d\right\}}{n} .
$$

Então $x$ será simplesmente normal se $\mathbb{P}(d)=10 \%$ para cada $d \in\{0,1, \ldots, 9\}$.

Dizemos que uma propriedade $P$ vale para quase todo ponto $x \in[0,1)$ se

$$
\{x \in[0,1) \mid P \text { é verdadeira }\}
$$

tiver medida total. O objetivo desta seção é entender a afirmação de que quase todo número $x \in[0,1)$ é simplesmente normal. Vamos precisar do seguinte resultado.

Teorema 11 (Teorema da equidistribuição). Seja $f:[0,1) \rightarrow[0,1)$ definida por $f(x)=\{10 x\}$. Sejam $x \in[0,1)$ um ponto e $\left(a_{n}\right)_{n=0}^{\infty}$ a sequência definida recursivamente por

$$
a_{0}=x, \quad a_{n}=f\left(a_{n-1}\right), n \geq 1 .
$$

Para quase todo $x \in[0,1)$ e para todo intervalo $J \subset[0,1)$, a sequência $\left(a_{n}\right)_{n=0}^{\infty}$ é equidistribuída em $J$, isto é,

$$
\lim _{n \rightarrow \infty} \frac{\#\left\{0 \leq k \leq n-1 \mid a_{k} \in J\right\}}{n}=|J|
$$

Demonstração. A prova deste resultado está fora do escopo deste texto. Trata-se de uma aplicação do Teorema Ergódico de Birkhoff. O resultado pode ser encontrado em [1, p. 2].

Teorema 12. Quase todo $x \in[0,1)$ é simplesmente normal, isto é, cada dígito $d \in\{0,1, \ldots, 9\}$ aparece com frequência de $10 \%$ na expansão decimal de $x$.

Demonstração. Seja $J=\left[\frac{d}{10}, \frac{d+1}{10}\right)$. Pelo Teorema 11 existe um conjunto $B \subset[0,1)$ de medida total tal que para todo $x \in[0,1)$, a sequência $a_{0}, a_{1}, \ldots$ definida por (2.10) é equidistribuída, isto é,

$$
\lim _{n \rightarrow \infty} \frac{\#\left\{0 \leq k \leq n-1 \mid \frac{d-1}{10} \leq a_{k}<\frac{d}{10}\right\}}{n}=\frac{d}{10}-\frac{d-1}{10}=\frac{1}{10}=10 \% .
$$

Sejam $x \in B$ e $x=0, d_{1} d_{2} \ldots$ a expansão decimal de $x$. Observe que

$$
\begin{aligned}
\left\{0 \leq k \leq n-1 \mid \frac{d-1}{10} \leq a_{k}<\frac{d}{10}\right\} & =\left\{0 \leq k \leq n-1 \mid d-1 \leq 10 a_{k}<d\right\} \\
& =\left\{0 \leq k \leq n-1 \mid\left\lfloor 10 a_{k}\right\rfloor=d\right\} \\
& =\left\{0 \leq k \leq n-1 \mid d_{k+1}=d\right\} . \\
& =\left\{1 \leq \ell \leq n \mid d_{\ell}=d\right\} .
\end{aligned}
$$


Desta forma,

$$
\mathbb{P}(d)=\lim _{n \rightarrow \infty} \frac{\#\left\{1 \leq \ell \leq n \mid d_{\ell}=d\right\}}{n}=\lim _{n \rightarrow \infty} \frac{\#\left\{0 \leq k \leq n-1 \mid \frac{d-1}{10} \leq a_{k}<\frac{d}{10}\right\}}{n}=10 \% .
$$



1. M. Einsiedler e T. Ward, Ergodic Theory with a view towards Number Theory, Springer, 2010, p. 2.

2. R. Nillsen, Randomness and recurrence in dynamical systems, The Carus Mathematical Monographs, Number 31, The Mathematical Association of America, pp. 92-106.

3. E. Süli e D. Mayers, An introduction to numerical analysis, Cambridge University Press, 2003, pp. 9-10.

4. A. Sultan e A. F. Artzt, The mathematics that every secondary school math teacher needs to know, Routledge, pp. 280-297. 